\title{
Connections for Health, Ecosystems and Society leading to Action and Change
}

The theme of connections has been integral to the field of ecohealth since its inception. At its core, ecohealth is about relationships and systemic interactions between issues, topics and approaches that are too often treated in isolation. As a central theme for EcoHealth 2014, the 5th biennial conference of the International Association for Ecology \& Health (IAEH), the emphasis on connections reminds us that we cannot reach our shared goal of ecosystem sustainability, resilience and healthfor humans and all species-alone. By focusing on the spaces in between, we are challenged to consider how the meeting of all forms of knowledge can not only increase understanding of complex problems affecting health, ecosystems and society, but also be mobilised to lead to actions to address these issues.

Arriving in Montreal in 2014 for an ecohealth gathering prompts reflection on how these confluences and convergences have developed and expanded 10 years since the Montreal EcoHealth Forum in 2003. One indication of this can be gleaned from the range of presenters assembling at the conference. At Ecohealth 2014, the programme not only includes researchers from natural, social and health sciences but also has very explicit inclusion of arts and other forms of practice, as well as representation of private and public sectors, NGOs and community groups. People from over 60 countries, spanning Africa, Asia, the Americas, Europe, Oceania and the Middle East, will present their work at the conference. Of particular note is the renewal and intergenerational spread, ranging from an increasingly strong presence of emerging scholars and practitioners, to an ongoing engagement with ecohealth pioneers.

Authors are members of Canadian Community of Practice in Ecosystem Approaches to Health (CoPEH-Canada).

Correspondence to: Johanne Saint-Charles, e-mail: saint-charles.johanne@uqam.ca
For those interested in connections, reflecting on developments since the 2003 Montreal EcoHealth Forum is less about defining progress and more about identifying consistent patterns of interactions and challenges that a field like ecohealth continues to face. In 2003, just as with all of the biennial conferences of the IAEH (Madison 2006, Merida 2008, London 2010, Kunming 2012), we assembled because of our need for integrative research, education and practice. The continuing excitement and dynamism of ecohealth arise not so much from any one area of expertise but in the fertile terrain of innovation that occurs when we come together to explore other ways of thinking and acting.

That anthropogenic environmental degradation impacts human and animal health is increasingly uncontroversial. Ecohealth scholars are tasked with both collecting and synthesizing information to better understand that core relationship. In addition, however, we face the more difficult task of defining what to do with that knowledge.

And so in 2014, we challenge ourselves to revisit and make explicit the important features of our ongoing aspiration towards connections. What can we learn from past connections? What types of conceptual and methodological connections are still needed to address the challenging domain that unites human and animal health, ecosystems and society? Whither the humanities as an integral contribution to ecohealth? EcoHealth2014 was proposed as a conference that will address these questions by contributing to 'the critical development of the field as well as to an international, interdisciplinary and intersectoral conversation about environment (natural and social) and health'. The motivation to leave the comfort of disciplinary or sectoral domains extends beyond interest in the richness of conversations or a commitment to the advancement of knowledge. Rather, we argue that interest in 
dialogue and connections among diverse stakeholders is stimulated by an imperative towards action and change, and that this imperative warrants explicit attention as ecohealth develops and refines its contributions in the coming decades.

A focus on ecohealth connections as pathways towards a world that prioritises social and gender equity, ecosystem sustainability and health for humans and other species, clearly positions us as agents of change and challenges the dogma of neutral science. We face many challenges in our quest to develop new approaches to research, education and practice that can accommodate different worldviews and prioritise dynamic interaction between research and action. When comparing ecohealth to other types of endeavours, we are now in a better position to identify some of the specific contributions arising from this field.

We propose that ecohealth's strong foundation within a complex system perspective has allowed for a better understanding of the importance of connections at multiple levels, with influences well beyond those who may identify as being 'part of ecohealth efforts. Key strengths and specificities of ecohealth lie in recognition of the emerging properties and new priorities that arise out of connections and also in willingness to engage with the uncertainties and ambiguities that are part of social-ecological systems. We also propose that trust is a basis for the relationships and connections that those working in ecohealth are inspired to build over the years, and that this trust is an essential component of the ability to traverse challenging terrain. This combination of willingness, trust-building and navigating uncertainty stands in stark contrast to the avoidance tactics of many traditions of research, education and practice that seek minimise or even "disappear" these important features of our complex world.

We also propose that ecohealth exhibits some particular intergenerational dynamics. Like other fields, there are genuine tensions as foundational pioneers are challenged and confronted by new and not so new ideas from a growing pool of emerging scholars and professionals and of newcomers to the field. However, we also anticipate that the skills and commitment to connections-including a sense of history and alertness to the need for iterations of learning and relearning — should offer strength and resilience for different features of the field as it develops to inform new approaches to and priorities for research, education, practice and action.

These specificities of ecohealth, we argue, make it particularly suited for action and change both requiring flexibility, adaptability and tolerance for doubt. These aptitudes and the associated commitment to connections are especially needed in a period where our planet, our collective home, is in a state of disarray at various levels; where humans, animals and ecosystems around the world are facing new and evolving challenges to their health and environmental and social justice seem still out of reach.

In essence, connections are active expressions of living and dynamic systems, and they carry with them a demand to be responsible for our actions. By refusing the disciplinary and sectoral dogmas, by insisting that our science and knowledge be confronted and criticised by other perspectives and worldviews, by asking that the research we do be anchored in communities' realities, we become active stewards of change.

Fostering connections not only demands us to think differently, but also to act differently, both as individuals and collectively. And that, for a field like ecohealth, will make all the difference.

Johanne Saint-Charles

Centre de recherche interdisciplinaire sur le bien-être, la santé, la société et l'environnement (Cinbiose), Université du Québec à Montréal, Montréal, QC, Canada

Département de communication sociale et publique, de l'Université du Québec à Montréal, Montréal, QC, Canada

Céline Surette

Environmental Studies Program,

Université de Moncton, Moncton, NB, Canada

Department of Chemistry and Biochemistry,

Université de Moncton, Moncton, NB, Canada

Margot W. Parkes

Canada Research Chair in Health, Ecosystems and Society, School of Health Sciences,

University of Northern British Columbia,

Prince George, BC, Canada

Northern Medical Program,

University of Northern British Columbia,

Prince George, BC, Canada

\section{Karen E. Morrison}

International Association for Ecology and Health, Guelph, ON, Canada

Faculty of Environmental Studies, York University, Toronto, ON, Canada

Published online: June 20, 2014 